\title{
Dark sulfur: Quantifying unpolymerized sulfur in inverse vulcanized polymers
}

\author{
Joseph J. Dale, Samuel Petcher and Tom Hasell* \\ Department of Chemistry, University of Liverpool, Crown Street, Liverpool L69 7ZD, UK E-mail: Loseph.Dale@liver- \\ pool.ac.uk, T. Hasell@liverpool.ac.uk
}

Received Address

\begin{abstract}
Elemental sulfur is produced as a by-product of the refining process of the petrochemicals industry. This generates an excess of sulfur each year that currently goes unused. A process known as inverse vulcanization allows polymeric materials to be formed from elemental sulfur, stabilised with organic comonomers or crosslinkers. The resultant high sulfur content polymers have shown many interesting and unique properties, and are being investigated for a growing number of applications. However, the techniques regularly used to determine if free unreacted sulfur, as $S_{8}$, remains in the materials only detect the crystalline, and not the amorphous form. Here is presented a detailed study on the identification and quantification of free amorphous sulfur present within inverse vulcanized polymers, both immediately after synthesis and after a period of aging, in which free sulfur is shown to increase over time. The potential for post-aging regeneration, by applying heat to stimulate homolytic disulphide cleavage, is also investigated.
\end{abstract}

Sulfur is a naturally occurring element that has been used by humankind for hundreds of years. Records of its use reach back to Roman times, in Pliny the Elder's discussions of minerology in his Naturalis Historia. ${ }^{1}$ Elemental sulfur has been employed for its antibacterial properties, treating ailments such as acne vulgaris, dermatitis, and warts. ${ }^{2-5}$ Indeed, Pliny refers (without evidence) to sulfurs use in the treatment of leprosy, asthma, and scorpion stings. In modern times however, the biggest use of sulfur is in the production of sulfuric acid, notably in the contact process. ${ }^{6,7}$ Sulfur itself is today produced mainly as a by-product of crude oil purification in the manufacture of petrochemicals. ${ }^{8}$ However, this process yields large quantities of sulfur in excess of that for which uses can currently be found. It is therefore desirable to identify new applications for sulfur to prevent the increasing buildup of waste, and in this regard Inverse Vulcanized Polymers may provide a solution. The seminal publication by Chung et al. introduced the process of Inverse Vulcanization ${ }^{9}$ in which sulfur is co-polymerized with a diene crosslinker to yield a polymeric product of high sulfur content (>50 wt.\%). Not only would utilization of excess sulfur be beneficial, but these novel materials possess a variety of properties, possessing demonstrable benefits in terms of antibacterial capacity, ${ }^{10,11}$ optics, ${ }^{12}$ mercury capture, ${ }^{13-15}$ and as encapsulating agents for fertilizer components. ${ }^{16}$ Significant interest has also attached to the use of these polymers in Li-S batteries ${ }^{17,18}$ due to their theoretical high energy storage capabilities (1762 mAhg-1). ${ }^{19}$ The different properties of sulfur-based polymer materials, including self-repair, ${ }^{20}$ high specific capacity, ${ }^{21,}{ }^{22}$ and tunable glass transition temperatures $\left(T_{\mathrm{g}}\right.$, the temperature above which the polymer transitions from a glass-like to an amorphous state), ${ }^{23}$ make them very attractive for a range of applications.
The variation in properties of these polymers can result from changes in synthesis conditions, sulfur loading, or crosslinker identity. ${ }^{23}$ Petrochemically derived molecules such as 1,3-diisopropenyl benzene (DIB), ${ }^{9}$ dicyclopentadiene (DCPD), ${ }^{14}$ and methyl styrene ${ }^{24}$ provide a range of crosslinked and linear polymers with highly varied physical characteristics. Such is the potential for variety in viable crosslinkers that renewable oils and plant-based derivatives such as canola oil ${ }^{25}$ or limonene ${ }^{26}$ can be used, enhancing the green credentials of this process.

Polymer aging is a major issue, ${ }^{27}$ and increasing interest in the development of deliberately purposed sulfur-based polymers necessitates improved understanding of the mechanisms of this aging. The $T_{\mathrm{g}}$ of a polymer is a key characteristic affecting properties such as mechanical strength and brittleness. ${ }^{28}$ While it has been shown that the $T_{\mathrm{g}}$ of these polymers is variable owing to reasons such as difference in crosslinker identity and synthesis conditions, it is shown here that this polymer property is subject to aging over time.

Much of the previously published work has assumed that the measure of a successful polymerisation, that is to say one in which all sulfur is polymerised and no free sulfur remains in the polymer, could be characterised by powder $\mathrm{x}$ ray diffraction (PXRD) or differential scanning calorimetry (DSC), concluding the absence of crystalline $\mathrm{S}_{8}{ }^{29-32}$ Whilst non-crystalline free sulfur has previously been observed in inverse vulcanized polymers, it has never been fully analysed or quantified. ${ }^{33}$ In order to quantify this free sulfur a high-performance liquid chromatography (HPLC) method has been developed allowing free sulfur to be extracted 
from the polymer. Here is reported an investigation of the aging, and unpolymerized amorphous $S_{8}$ content, of a range of inverse vulcanized polymers, as well as the potential to regenerate their properties. This amorphous $\mathrm{S}_{8}$ is referred to as 'dark sulfur' - amorphous sulfur that is not observable by preestablished analytical techniques.

(a)

(b)

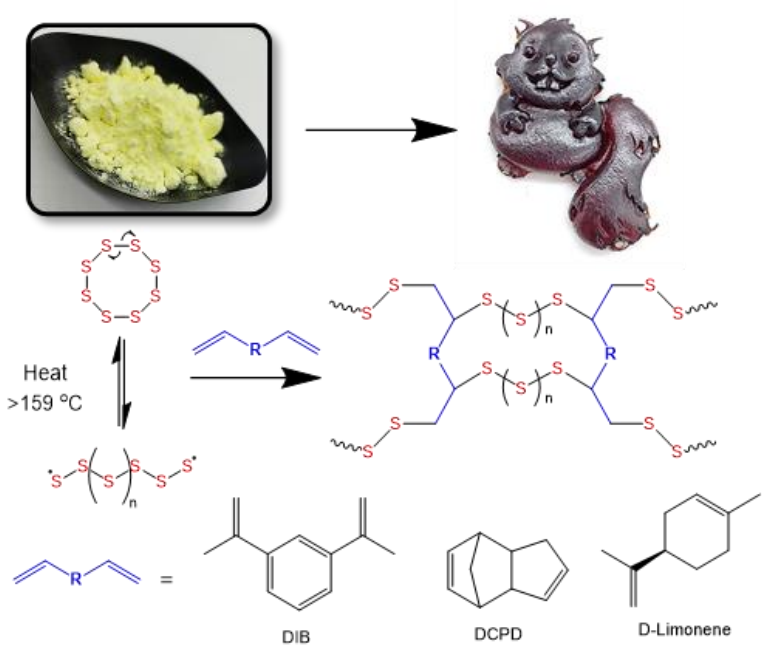

Figure 1. (a) The conversion of elemental sulfur to a polyS-crosslinker polymer material (b) The reaction mechanism of elemental sulfur reacting with a generic crosslinker molecule to form a sulfur based polymer via inverse vulcanization (c) The chemical structures of 1,3-diisopropenyl benzene (DIB), dicyclopentadiene (DCPD), and D-Limonene, the 3 main crosslinkers considered in this work.

Sulfur polymers were synthesised by the reaction of $\mathrm{S}_{8}$ and organic comonomer in equal mass at $170^{\circ} \mathrm{C}$ under agitation at $600 \mathrm{rpm}$. Upon reaching homogeneity, this polymer solution was oven cured at $140{ }^{\circ} \mathrm{C}$ for 24 hours. See SI (Figure S1) for more details. During our investigations into polymer aging, it was observed that the $T_{\mathrm{g}}$ of the assessed polymers increased over time, except for S-DCPD (Figure 1). No loss of mass was noted, nor an increase in crystalline sulfur (measured by DSC) unless crystalline sulfur was present upon the commencement of aging (Figure S2-6). After aging for six months (with changes in room temperature monitored for 30 days during this period (Figure S7)), all polymers remained insoluble, with no alterations in the infrared spectra (FTIR) suggesting that no structural change in the organic moieties was taking place, or the formation of such species as thiols (Figure S8-10). This suggests that there may be a change in sulfur rank over time. The sulfur rank (the number of sulfur atoms per chain) may be variable across the polymer upon synthesis, and consists of a number of weaker S-S linkages end capped by stronger C-S bonds. The longer the sulfur chain length, the weaker the S$S$ linkage towards the centre of the chain. Over time these weak bonds in the middle of the chain may naturally cleave, liberating sulfur into the polymer matrix. The polymer chain may then reform, thus maintaining the degree of crosslinking. (a)

(b)
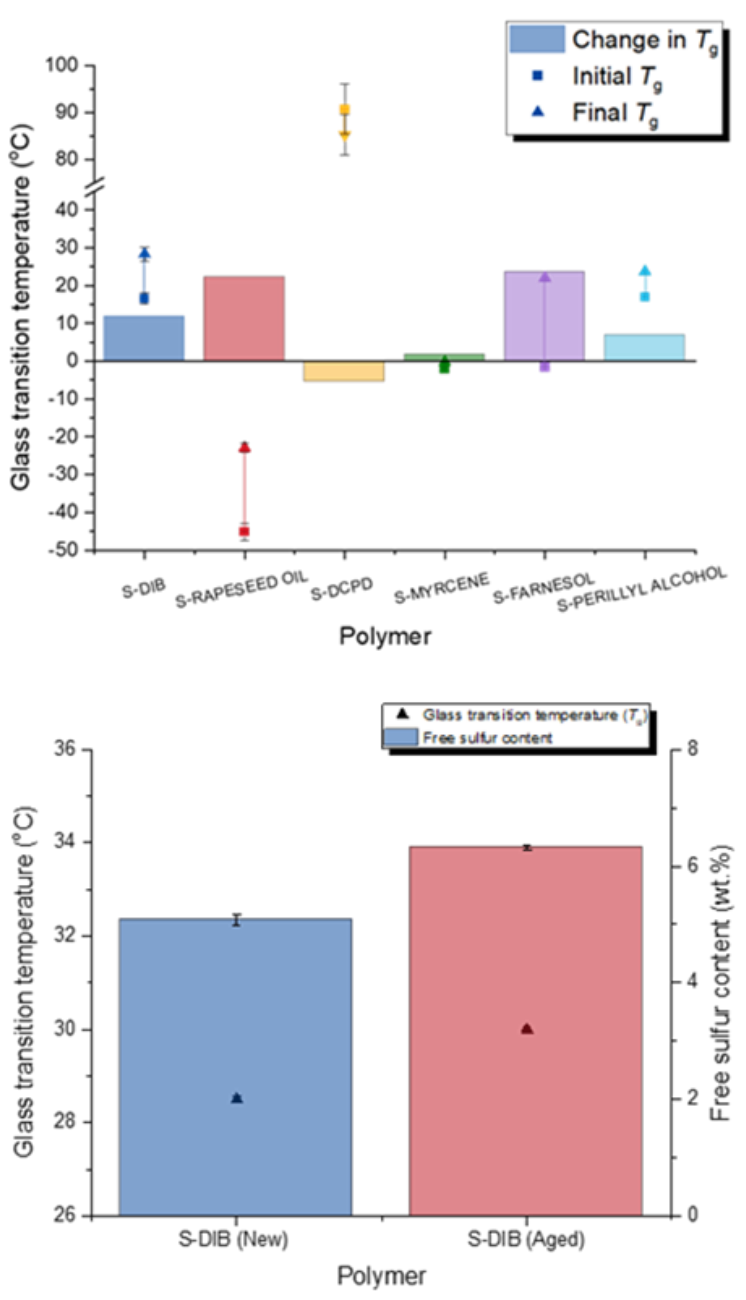

Figure 2. (a) The change in $T_{\mathrm{g}}$ of a range of polymers, selected for having a range of initial values of $T_{\mathrm{g}}$. Polymers were synthesised with a sulfur loading of $50 \mathrm{wt} . \%$ to 50 wt. $\%$ comonomer. Aging time $=6$ months (b) A comparison between aged and new S-DIB, showing an increase in both $T_{\mathrm{g}}$ and dark sulfur content (from HPLC). Aging time $=4$ months.

This reformation leads to the observed increase in $T_{\mathrm{g}}$, as the linear sections become shorter. To confirm the existence of dark sulfur, polymer samples were analysed by thin layer chromatography (TLC) and HPLC. Solvent extraction of free sulfur was carried out in toluene, with solutions at concentrations of $5 \mathrm{mg} \mathrm{mL}-1$ prepared, and passed through $25 \mathrm{~mm}$ $0.45 \mu \mathrm{m}$ nylon syringe filters. TLC analysis was carried out in a hexane mobile phase. This suggested that, while no free crosslinker was present in the polymer samples, unpolymerized sulfur was present in both new and aged polymers, despite no crystalline sulfur having been observed in the DSC analysis (Figure S11) A method for analysis of elemental sulfur by HPLC was then developed, adapted from the method detailed by Buchanan et al. for the analysis of free sulfur in coal. ${ }^{34}$ Elemental sulfur in the form of $S_{8}$ is eluted from the column at a residence time of 7.3 minutes (Figure S12-13). It should be noted that the temperature 
increase in the HPLC analysis may lead to the formation of further polysulfides, so that if oligomeric species were extracted into the solvent with the dark sulfur the quantity of sulfur observed may be slightly greater than the real value. Comparison of both aged and new S-DIB confirmed an increase in the quantity of free sulfur of $1.2 \mathrm{wt} . \%$ in a polymer with a sulfur loading of $50 \mathrm{wt} \%$ after a period of 4 months (Figure 2). Analysis of the new S-DIB was conducted immediately after synthesis.

(a)

(b)
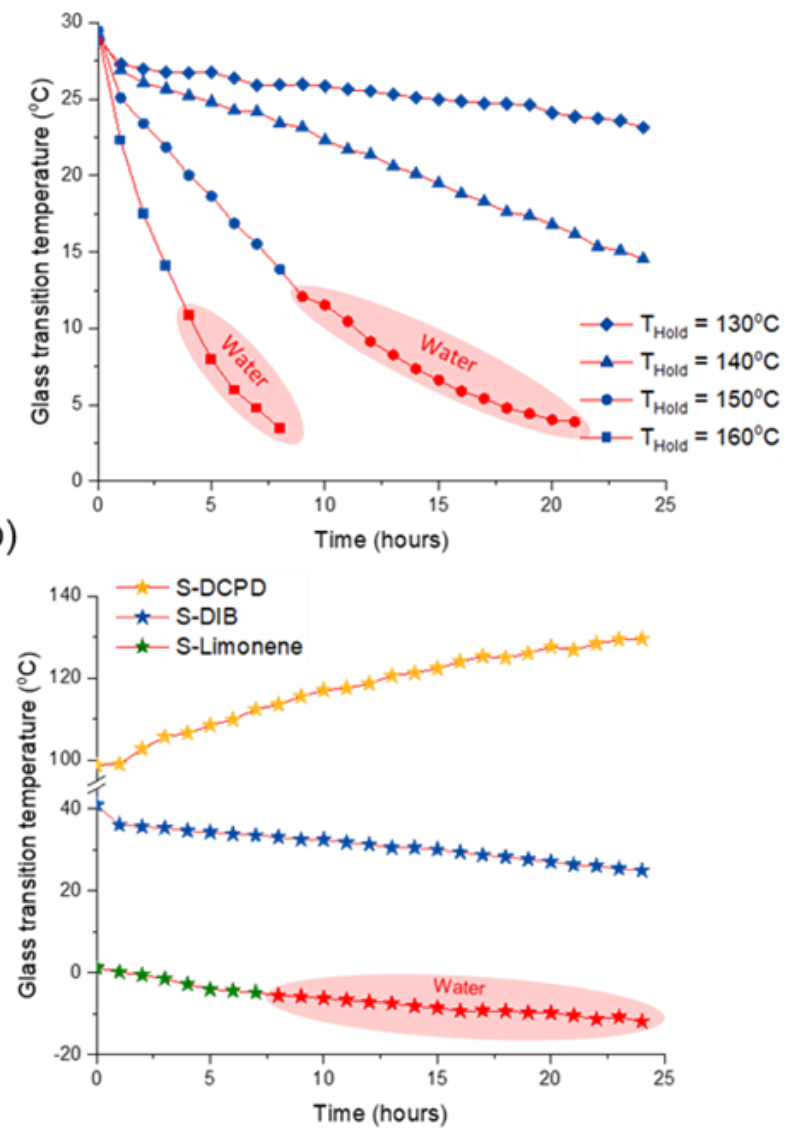

Figure 3. The change in $T_{\mathrm{g}}$ with re-curing of (a) an aged sample of S-DIB at hold temperatures of $130-160{ }^{\circ} \mathrm{C}$ (b) newly synthesised samples of S-DCPD, S-DIB, and S-Limonene at a hold temperature of $140{ }^{\circ} \mathrm{C}$. Water appears in the DSC traces of certain samples and conditions, suggesting the polymer chains are mobile enough to allow the release of trapped moisture.

This coincides with an increase in glass transition temperature of $1.5^{\circ} \mathrm{C}$ over this time. This contributes to the suggestion that sulfur may be liberated from the sulfur chains, shortening the sulfur rank and thus increasing the $T_{\mathrm{g}}$. This conclusion regarding polymer aging has significance for the development and consideration of inverse vulcanized polymers, and further research is being carried out to fully nderstand how aging may affect polymer properties and application performance.
It may be possible to reverse this aging due to the dynamic nature of the S-S bonds in the longer polymer chains. The application of heat may allow for homolytic S-S cleavage and subsequent re-insertion of this 'dark' sulfur into the polymer chains. This would result in a predicted decrease in the $T_{\mathrm{g}}$ and dark sulfur content. An experiment was therefore designed using DSC, whereby $5 \mathrm{mg}$ of a polymer was recured by heating to a designated temperature and held for 1 hour inside the DSC, characterised by temperature sweep, and then similarly cured further. This was repeated over a 25 hour period (the first hour allowing the sample to reach thermal equilibrium). It was observed that upon heating the $T_{\mathrm{g}}$ decreased linearly, with higher hold temperatures yielding a faster rate of $T_{\mathrm{g}}$ decrease (Figure 3 ).

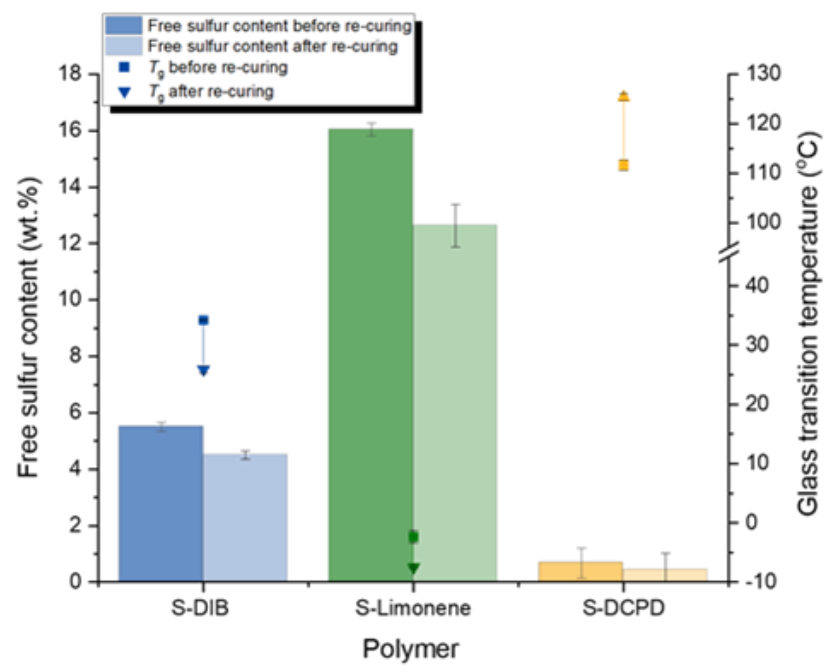

Figure 4. The change in $T_{\mathrm{g}}$ and free sulfur contents of newly synthesised S-DIB, S-Limonene, and S-DCPD after re-curing for 24 hours at $140{ }^{\circ} \mathrm{C}$ in sealed containers.

As it was observed that new samples of S-DIB also contain dark sulfur, this experiment was repeated for newly synthesised samples of S-DCPD, S-DIB, and S-Limonene, chosen to represent polymers of a wide range of initial $T_{\mathrm{g}}$ and sulfur rank. S-DIB and S-Limonene showed a decrease in $T_{\mathrm{g}}$ suggesting the reinsertion of sulfur into the polymer chains. SDCPD showed an increase in $T_{\mathrm{g}}$. The average estimated sulfur rank of S-DCPD is 1.94, thus suggesting the polymer is made up of a series of strong disulfide linkages. The strength of these disulfides is such that the bonds may not homolytically cleave, therefore preventing the structural rearrangement of the polymer, and elongation of the polymer chains. It is predicted that S-DIB ${ }^{35}$ and S-Limonene contain longer polysulfide chains with weaker S-S bonds that may cleave under applied heat, allowing for rearrangement (Figure S14). It must also be noted that water began to accumulate in the DSC traces after a period of time, obscuring the $T_{\mathrm{g}}$ of some samples. This water originates from within the polymer, where it has accumulated over the course of the aging period from adventitious moisture (Figure S15-16). The application of heat allows for increased mobility of the elongating polymer chains and the water to escape the polymer. Water appears in the new sample of S-Limonene as it has a $T_{\mathrm{g}}$ lower than room temperature, therefore existing in a 
form where the polymer chains are more mobile, allowing for the entrance of moisture rapidly into the sample.

To analyse a change in dark sulfur with re-curing, a series of polymers of S-DCPD, S-DIB, and S-Limonene were held in an oven at $140{ }^{\circ} \mathrm{C}$ for 24 hours. After this period of re-curing the $T_{\mathrm{g}}$ of all polymers increased (Figure S17). As the polymers were open to the air, it was possible for the dark sulfur to migrate to the surface and escape the polymer, further evidenced by the appearance of sulfur crystals in the extraction vents of the oven. The polymers were then able to cure further and rearrange into a more crosslinked structure. This investigation was repeated with the polymer samples contained in sealed DSC pans. It was subsequently observed that the $T_{\mathrm{g}}$ of the sealed polymers decreased with a decrease in dark sulfur (figure 4), in agreement with the data presented in Figure 3. Once again S-DCPD shows an increase in $T_{\mathrm{g}}$, correlating with further curing of the polymer and the inability to break the sulfur chain linkages. Little difference in re-curing was observed with alterations in gas flow over the sample, thus confirming the re-curing effect is derived from the application of heat (Figure S18). This data suggests that inverse vulcanized polymers may boast regenerative capabilities, with the potential to reverse the aging that may occur during the polymer lifetime.

In summary, it has been determined that the aging of inverse vulcanized polymers is not simply limited to changes observable by the eye. This investigation into polymer aging has confirmed the existence of free amorphous $\mathrm{S}_{8}$ in both new and aged polymers showing that, even in the absence of crystalline sulfur, a polymerization may not be considered as complete. There is a possibility that this sulfur may be incorporated back into the polymer chains through the application of heat, in a sealed system, above the melting point of sulfur. This free sulfur was quantified by HPLC, though the authors accept the limitations of this method, such as the possibility that the solvent extraction method may itself affect the polymers. Independent analyses and further research are currently being conducted to assess the effects of dark sulfur on polymer properties, and determine other methods of analysis. We suggest this area of discovery may benefit from further investigation into other analytical methods for analysis of amorphous sulfur, ideally in-situ.

\section{ASSOCIATED CONTENT}

Sample preparation, DSC, storage temperature analysis, FTIR, TLC, HPLC, predicted average sulfur ranks of polymers. This material is available free of charge via the Internet at http://pubs.acs.org.

\section{AUTHOR INFORMATION}

Corresponding Author

* Tom Hasell - Department of Chemistry, University of Liverpool, Crown Street, Liverpool L69 7ZD, UK E-mail: T. Hasell@liverpool.ac.uk

\section{Authors}

Joseph J. Dale - Department of Chemistry, University of Liverpool, Crown Street, Liverpool L69 7ZD, UK

Samuel Petcher - Department of Chemistry, University of Liverpool, Crown Street, Liverpool L69 7ZD, UK

\section{Notes}

The authors declare no conflict of interest.

\section{Acknowledgements}

We thank K. Farrance for assistance in HPLC method development. TH is a Royal Society University Research Fellow.

\section{REFERENCES}

1. Murphy, T. M., Pliny the Elder's Natural History: The Empire in the encyclopaedia Oxford: Oxford University Press: Oxford, 2004.

2. Inoue, H.; Kawano, G.; Nagasawa, H.; Sakuda, S., Isolation of elemental sulfur as a self-growth-inhibiting substance produced by Legionella pneumophila. Applied and Environmental Microbiology 2002, 68 (10), 4809-4811.

3. Lawson, G. B., The Inhibitory Action of Sulphur on the Growth of Tubercle Bacilli. American Review of Tuberculosis 1934, 29 (6), 650-651.

4. Weld, J. T.; Gunther, A., THE ANTIBACTERIAL PROPERTIES OF SULFUR. Journal of Experimental Medicine 1947,85 (5), 531-542.

5. Gupta, A. K.; Nicol, K., The use of sulfur in dermatology. Journal of Drugs in Dermatology 2004, 3 (4), 427 431.

6. Chenier, P. J., Survey of Industrial Chemistry John Wiley \& Sons: New York, 1987.

7. Moeller, W.; Winkler, K., The Double Contact Process for Sulfuric Acid Production. Journal of the Air Pollution Control Association 1968, 18 (5), 324-325.

8. Rauchfuss, T., Under sulfur's spell. Nature Chemistry 2011, 3 (8), 648-648.

9. Chung, W. J.; Griebel, J. J.; Kim, E. T.; Yoon, H.; Simmonds, A. G.; Ji, H. J.; Dirlam, P. T.; Glass, R. S.; Wie, J. J.; Nguyen, N. A.; Guralnick, B. W.; Park, J.; Somogyi, A.; Theato, P.; Mackay, M. E.; Sung, Y. E.; Char, K.; Pyun, J., The use of elemental sulfur as an alternative feedstock for polymeric materials. Nature Chemistry 2013, 5 (6), 518-524. 
10. Smith, J. A.; Mulhall, R.; Goodman, S.; Fleming, G.; Allison, H.; Raval, R.; Hasell, T., Investigating the Antibacterial Properties of Inverse Vulcanized Sulfur Polymers. Acs Omega 2020, 5 (10), 5229-5234.

11. Deng, Z. L.; Hoefling, A.; Theato, P.; Lienkamp, K., Surface Properties and Antimicrobial Activity of Poly(sulfur-co-1,3-diisopropenylbenzene) Copolymers. Macromolecular Chemistry and Physics 2018, 219 (5).

12. Wadi, V. S.; Jena, K. K.; Halique, K.; Rozic, B.; Cmok, L.; Tzitzios, V.; Alhassan, S. M., Scalable High Refractive Index polystyrene-sulfur nanocomposites via in situ inverse vulcanization. Scientific Reports 2020, 10 (1).

13. Lee, J. S. M.; Parker, D. J.; Cooper, A. I.; Hasell, T., High surface area sulfur-doped microporous carbons from inverse vulcanised polymers. Journal of Materials Chemistry A 2017, 5 (35), 18603-18609.

14. Parker, D. J.; Jones, H. A.; Petcher, S.; Cervini, L.; Griffin, J. M.; Akhtar, R.; Hasell, T., Low cost and renewable sulfur-polymers by inverse vulcanisation, and their potential for mercury capture. Journal of Materials Chemistry A 2017, 5 (23), 11682-11692.

15. Hasell, T.; Parker, D. J.; Jones, H. A.; McAllister, T.; Howdle, S. M., Porous inverse vulcanised polymers for mercury capture. Chemical Communications 2016, 52 (31), 5383-5386.

16. Mann, M.; Kruger, J. E.; Andari, F.; McErlean, J.; Gascooke, J. R.; Smith, J. A.; Worthington, M. J. H.; McKinley, C. C. C.; Campbell, J. A.; Lewis, D. A.; Hasell, T.; Perkins, M. V.; Chalker, J. M., Sulfur polymer composites as controlledrelease fertilisers. Organic \& Biomolecular Chemistry 2019, 17 (7), 1929-1936.

17. Simmonds, A. G.; Griebel, J. J.; Park, J.; Kim, K. R.; Chung, W. J.; Oleshko, V. P.; Kim, J.; Kim, E. T.; Glass, R. S.; Soles, C. L.; Sung, Y. E.; Char, K.; Pyun, J., Inverse Vulcanization of Elemental Sulfur to Prepare Polymeric Electrode Materials for Li-S Batteries. Acs Macro Letters 2014, 3 (3), 229232.

18. Zhang, Y. Y.; Griebel, J. J.; Dirlam, P. T.; Nguyen, N. A.; Glass, R. S.; Mackay, M. E.; Char, K.; Pyun, J., Inverse vulcanization of elemental sulfur and styrene for polymeric cathodes in Li-S batteries. Journal of Polymer Science Part a-Polymer Chemistry 2017, 55 (1), 107-116.

19. Gomez, I.; Leonet, O.; Blazquez, J. A.; Mecerreyes, D., Inverse Vulcanization of Sulfur using Natural Dienes as Sustainable Materials for Lithium-Sulfur Batteries. Chemsuschem 2016, 9 (24), 3419-3425.

20. Tonkin, S. J.; Gibson, C. T.; Campbell, J.; Lewis, D.; Karton, A.; Hasell, T.; Chalker, J. M., Chemically induced repair, adhesion, and recycling of polymers made by inverse vulcanization. Chemical Science 2020, 11 (21), 5537-5546.
21. Li, X.; Yuan, L. X.; Liu, D. Z.; Li, Z.; Chen, J.; Yuan, K.; Xiang, J. W.; Huang, Y. H., High sulfur-containing organosulfur polymer composite cathode embedded by monoclinic $\mathrm{S}$ for lithium sulfur batteries. Energy Storage Materials 2020, 26, 570-576.

22. Liu, J.; Wang, M. F.; Xu, N.; Qian, T.; Yan, C. L., Progress and perspective of organosulfur polymers as cathode materials for advanced lithium-sulfur batteries. Energy Storage Materials 2018, 15, 53-64.

23. Smith, J. A.; Wu, X. F.; Berry, N. G.; Hasell, T., High Sulfur Content Polymers: The Effect of Crosslinker Structure on Inverse Vulcanization. Journal of Polymer Science Part a-Polymer Chemistry 2018, 56 (16), 1777-1781.

24. Martin, T. R.; Mazzio, K. A.; Hillhouse, H. W.; Luscombe, C. K., Sulfur copolymer for the direct synthesis of ligand-free $\mathrm{CdS}$ nanoparticles. Chemical Communications 2015, 51 (56), 11244-11247.

25. Lundquist, N. A.; Worthington, M. J. H.; Adamson, N.; Gibson, C. T.; Johnston, M. R.; Ellis, A. V.; Chalker, J. M., Polysulfides made from re-purposed waste are sustainable materials for removing iron from water. Rsc Advances 2018, 8 (3), 1232-1236.

26. Crockett, M. P.; Evans, A. M.; Worthington, M. J. H.; Albuquerque, I. S.; Slattery, A. D.; Gibson, C. T.; Campbell, J. A.; Lewis, D. A.; Bernardes, G. J. L.; Chalker, J. M., Sulfur-Limonene Polysulfide: A Material Synthesized Entirely from Industrial By-Products and Its Use in Removing Toxic Metals from Water and Soil. Angewandte Chemie-International Edition 2016, 55 (5), 1714-1718.

27. Hutchinson, J. M., PHYSICAL AGING OF POLYMERS. Progress in Polymer Science 1995, 20 (4), 703-760.

28. Yan, P. Y.; Zhao, W.; Zhang, B. W.; Jiang, L.; Petcher, S.; Smith, J. A.; Parker, D. J.; Cooper, A. I.; Lei, J. X.; Hasell, T., Inverse Vulcanized Polymers with Shape Memory, Enhanced Mechanical Properties, and Vitrimer Behavior. Angewandte Chemie-International Edition 2020, 59 (32), 13371-13378.

29. Arslan, M.; Kiskan, B.; Yagci, Y., Combining Elemental Sulfur with Polybenzoxazines via Inverse Vulcanization. Macromolecules 2016, 49 (3), 767-773.

30. Wu, F. X.; Chen, S. Q.; Srot, V.; Huang, Y. Y.; Sinha, S. K.; van Aken, P. A.; Maier, J.; Yu, Y., A Sulfur-LimoneneBased Electrode for Lithium-Sulfur Batteries: High-Performance by Self-Protection. Advanced Materials 2018, 30 (13).

31. Valle, S. F.; Giroto, A. S.; Klaic, R.; Guimaraes, G. G. F.; Ribeiro, C., Sulfur fertilizer based on inverse vulcanization process with soybean oil. Polymer Degradation and Stability 2019, 162, 102-105.

32. Lee, J. M.; Noh, G. Y.; Kim, B. G.; Yoo, Y.; Choi, W. J.; Kim, D. G.; Yoon, H. G.; Kim, Y. S., Synthesis of 
Poly(phenylene polysulfide) Networks from Elemental Sulfur and p-Diiodobenzene for Stretchable, Healable, and Reprocessable Infrared Optical Applications. Acs Macro Letters 2019, 8 (8), 912-916.

33. Najmah, I. B.; Lundquist, N. A.; Stanfield, M. K.; Stojcevski, F.; Campbell, J. A.; Esdaile, L. J.; Gibson, C. T.; Lewis, D. A.; Henderson, L. C.; Hasell, T.; Chalker, J. M., Insulating Composites Made from Sulfur, Canola Oil, and Wool**. Chemsuschem 2021, 14 (11), 2352-2359.
34. Buchanan, D. H.; Coombs, K. J.; Murphy, P. M., Energy and Fuels 1993, (7), 219-221.

35. Kiani, R.; Sebastiani, D.; Partovi-Azar, P., On the structure of sulfur/1,3-diisopropenylbenzene co-polymer cathodes for Li-S batteries: insights from density-functional theory calculations. Chemphyschem. 